\title{
The Short-Run Effects of Unanticipated Monetary Shocks under Distinct Trading Mechanisms
}

\author{
Luis Araujo, Michigan State University and \\ Sao Paulo School of Economics - FGV \\ araujolu@msu.edu \\ Andrei Shevchenko, Michigan State University \\ shevchen@msu.edu
}

We unveil the existence of a trade-off between efficiency and information transmission in a decentralized economy subject to a monetary shock. If the objective is to maximize information transmission, then the optimal trading protocol is inherently inefficient. If, instead, the objective is to maximize efficiency, then the optimal trading protocol is necessarily uninformative.

Keywords: monetary uncertainty, Citation: Araujo, L. and search, information transmission, Shevchenko, A. (2018). The Shorttrading protocols

JEL Codes: E40, D82, D83
Run Effects of Unanticipated Monetary Shocks under Distinct Trading Mechanisms. Russian Journal of Money and Finance, 77(3), pp. 76-88.

doi: $10.31477 /$ rjmf.201803.76

\section{Introduction}

In this paper we study two alternative price protocols and show how they influence production and prices in the short run under monetary uncertainty. The first protocol is akin to price posting, while the second one is akin to bargaining. These protocols differ in the amount of information they potentially transfer between buyers and sellers. Intuitively, compared to price posting, bargaining provides more instruments for market participants to learn about the actual state of the economy. We want to explore whether this learning enhances or hinders efficiency.

The literature on the short run effects of monetary uncertainty is vast, starting with the seminal paper by Lucas (1972). For the most past, this literature concentrates on price taking (e.g., Faig and Li, 2009) or price posting (e.g., Golosov and Lucas, 2007) as the trading mechanisms, but various papers also consider bargaining (e.g., Wallace, 1997; Katzman et al., 2003; Araujo and Shevchenko, 
2006; Hu and Wallace, 2016). As far as we know, there is no paper that considers price posting and bargaining in the same environment and explores the ensuing trade-off between efficiency and information transmission.

Our baseline environment follows Trejos and Wright (1995) and Wallace (1997), a decentralized framework that allows to introduce uncertainty in an attractive way. We assume that the economy experiences a one time monetary injection and look at the implications of this shock in terms of efficiency and information transmission. Our main result is that a trading protocol that resembles price posting is efficient but uninformative, while a trading protocol that resembles bargaining is informative but inefficient. In particular, in contrast to the view that price dispersion is a manifestation of confusion about the true state of the economy (e.g., Stigler, 1961), we argue that price volatility is the result of a beneficial "experimentation" by buyers and sellers.

A paper that is related to our work is Camera and Delacroix (2004). They endogenize the trading mechanism by allowing sellers to choose between price posting and haggling, trading off the probability of an immediate sale versus expected surplus. This trade off leads to implications that are different from our results. In their model under price posting sellers do not trade with everyone, while the haggling regime may generate trading delays.

Our environment is stylized but we believe that it sheds some light on the dynamics of important markets. An example of a market that is in a constant state of flux is the overnight Federal Funds market. In this market the reserve position of each bank changes constantly due to a large number of random transactions initiated by depositors during the day. By the end of the day the bank wants to readjust its reserve position to be in compliance with the Federal Reserve regulations and at the same time maximize its profits. Many studies show that the peak of trading activity in this market is concentrated in the last two hours of the trading day (which is about $40 \%$ of total trading, according to Bartolini et al., 2005). During this period, the larger share of all transactions are arranged directly and bilaterally between banks, and an interesting fact is that usually depository institutions conduct several subsequent transactions during the 2-hour end-ofthe-day window. Instead of just borrowing or lending a needed amount in one transaction, managers make several subsequent calls, every time bargaining over both the amount of the loan and the rate. This suggests that learning about the actual value of money is an important component. In fact, Afonso and Lagos (2015) is a recent paper which provides microfoundations of the Federal Funds market while taking seriously search frictions, random bilateral meetings, and bargaining. At the end of their paper, the authors suggest as a "natural extension" the incorporation of private information into the analysis.

The rest of the paper proceeds as follows. The next section describes the model. Then we consider two trading protocols and we characterize equilibria under each protocol. Section 3 concludes the paper. 


\section{Model}

Our model is a modified version of Wallace (1997). Consider an economy with a continuum of agents and $N$ divisible and perishable goods. Time is discrete and indexed by $t$. Agents can be of $N$ distinct types. A type $i$ agent derives utility from the consumption of good $i$ and is able to produce good $i+1$ (modulo $N$ ). Agents are uniformly distributed across types and maximize expected discounted utility with a discount factor $\beta$. Utility in every period is given by $u(x)-y$ where $x$ is the quantity consumed and $y$ is the quantity produced. The function $u$ is defined on $[0, \infty)$ and $u($.$) is increasing, twice differentiable and strictly concave. There is a$ storable and indivisible object, which we denote as fiat money. We assume that an agent can hold at most one unit of money at a time.

Trade is decentralized, with frictions in the exchange process. Precisely, there are $N$ distinct sectors, each sector specialized in the exchange of one good. Agents can identify sectors, but inside a sector they are anonymous and matched pairwise under a uniform random matching technology. Each agent faces one meeting per period. Moreover, meetings are independent across agents and independent over time. Therefore, if an agent wants money he goes to the sector which trades his endowment and searches for an agent with money. If he has money, he goes to the sector that trades the good he likes and searches for an agent with the good. Since there is an upper bound on money holdings, a transaction can happen only when an agent with money (buyer) meets an agent without money (seller). We denote meetings of this sort as trade meetings.

The quantity of money in the economy at the end of date 0 is equal to $m$, and it is uniformly distributed across types. At the beginning of date 1, there is a onetime increase in the quantity of money. This increase comes as a random draw from a commonly known distribution $P(\Delta)$, with $P\left(\Delta=\Delta_{k}\right)=1 / 2, k \in\{L, H\}$. We assume that $1-m>\Delta_{H}>\Delta_{L} \geq 0$. Given $\Delta=\Delta_{k}$, an agent who leaves the economy without money at date 0 receives money with a probability $\frac{\Delta_{k}}{1-m}$. During date 1 ,

the actual value of $\Delta$ is not observable. However, agents can use their private histories to update beliefs with respect to the value of money. At the beginning of date 2 , before meetings take place, the quantity of money is revealed to all agents.

In every meeting between a buyer and a seller, the buyer makes a take it or leave it offer to the seller. We consider two distinct cases, which vary according to the informativeness of the offer with respect to buyer's belief about the state of the economy. In the first case, which we label "price posting", the seller first posts the quantity $q$ of the good that he wants to sell, and then the buyer proposes a probability $\tau \in[0,1]$ with which he will transfer the money in exchange for the quantity $q$. Trade occurs if the seller accepts the offer. In the second case, which we label "bargaining", the buyer proposes a pair $(\tau, q) \in[0,1] \times \mathbb{R}^{+}$. If the seller accepts the proposal, he produces $q$ and money changes hands with probability $\tau$. 
Discussion. Our main objective is to examine how trading protocols affect efficiency and information transmission in a decentralized economy. There are many distinct trading protocols that one could think of, and it is not without loss of generality that we restrict attention to the two protocols described above. However, we believe that our choice captures important elements in the process of exchange. Precisely, the protocol where the seller first commits to produce some quantity and the buyer can only choose the probability that he will transfer the unit of money, captures instances where the buyer does not have much room to communicate his beliefs about the underlying value of money. We think of this case as informationally equivalent to a price posting scheme where the seller announces a price $p=\frac{\tau}{q}$ and the buyer decides how much to buy of the good. In turn, the protocol where the buyer can announce not only the probability that he will transfer money, but also the specific quantity of the good that he wants in return, captures instances where there is more room for communication between the buyer and the seller. We like to think of this case as capturing features of a bargaining scheme.

\subsection{Equilibrium}

We solve the model by backward induction. Consider the economy at the beginning of date $t>1$. Let us consider first the scenario where the set of possible offers is given by $(\tau, q) \in[0,1] \times \mathbb{R}^{+}$. The assumption that buyers make a take it or leave it offer to sellers implies that the seller must be indifferent between accepting or rejecting the offer. Precisely,

$$
q_{k}=\beta \tau_{k}\left[V_{1}\left(\Delta_{k}\right)-V_{0}\left(\Delta_{k}\right)\right],
$$

where $V_{1}\left(\Delta_{k}\right)$ is the value of holding money when the quantity of money is equal to $m+\Delta_{k}$, and $V_{0}\left(\Delta_{k}\right)$ is the value of not holding money when the quantity of money is equal to $m+\Delta_{k}$. Take it or leave it offers implies that $V_{0}\left(\Delta_{k}\right)=0$ and

$$
V_{1}\left(\Delta_{k}\right)=\left(m+\Delta_{k}\right) \beta V_{1}\left(\Delta_{k}\right)+\left(1-m-\Delta_{k}\right)\left[u\left(q_{k}\right)+\left(1-\tau_{k}\right) \beta V_{1}\left(\Delta_{k}\right)\right] \text {. }
$$

Substituting (1) in (2), we obtain

$$
V_{1}\left(\Delta_{k}\right)=\frac{\left(1-m-\Delta_{k}\right)\left[u\left(q_{k}\right)-q_{k}\right]}{1-\beta} .
$$

If the buyer makes an offer that the seller accepts, his payoff is

$$
u\left(q_{k}\right)+\left(1-\tau_{k}\right) \beta V_{1}\left(\Delta_{k}\right)=u\left(q_{k}\right)-q_{k}+\beta V_{1}\left(\Delta_{k}\right) .
$$

The unconstrained choice of $q_{k}$ that maximizes the buyer's payoff is $q_{k}=q^{*}$, and the corresponding transfer is

$$
\tau_{k}=\frac{1-\beta}{\beta} \frac{q^{*}}{u\left(q^{*}\right)-q^{*}} \frac{1}{1-m-\Delta_{k}} .
$$


Finally, since $\tau_{k} \in[0,1]$, the unconstrained choice of $q_{k}$ is feasible if and only if $\tau_{k} \leq 1$, that is

$$
\frac{1-\beta}{\beta} \frac{q^{*}}{u\left(q^{*}\right)-q^{*}} \frac{1}{1-m-\Delta_{k}} \leq 1
$$

Throughout the paper, we assume that (4) always holds. Thus, whenever agents have complete information about the state of the economy, the equilibrium consists of producing the efficient quantity $q^{*}$ and making a transfer $\tau_{k}$.

Before working backwards to date 1 , we need to consider the scenario where the seller first commits to produce a given quantity and then the buyer proposes a transfer. It should be clear though that, in this case, there exists an equilibrium implies the same outcome as in the scenario where the set of possible offers is given by $(\tau, q) \in[0,1] \times \mathbb{R}^{+}$. Indeed, if the seller commits to produce $q^{*}$, it is best for the buyer to propose to transfer money with probability $\tau_{k}$, a proposal which is accepted by the seller.

At date 1, since the amount of money in the economy is not known, the equilibrium outcome will depend on the trading protocol. We start with the scenario where the seller first commits to a given quantity $q$ and then the buyer chooses a transfer $\tau \in[0,1]$. We label this case as price posting. We then consider the scenario where the set of possible offers by the buyer is given by $(\tau, q) \in[0,1] \times \mathbb{R}^{+}$. We label this case as bargaining.

\subsubsection{Price posting}

Consider a trade meeting at date 1 . All sellers in these meetings had the same experience: they started with no money at the end of date 0 , received no money at the beginning of date 1 and met an agent with money during date 1 . Given this history, sellers form posteriors about the state of the economy. Precisely, if we define $\Theta_{S}$ as the seller's posterior belief that the quantity of money is equal to $m+\Delta_{L}$, we obtain

$$
\Theta_{S}=\frac{\left(1-m-\Delta_{L}\right)\left(m+\Delta_{L}\right)}{\left(1-m-\Delta_{L}\right)\left(m+\Delta_{L}\right)+\left(1-m-\Delta_{H}\right)\left(m+\Delta_{H}\right)} .
$$

Consider now the histories of buyers. Some buyers are carrying new money injected at the beginning of date 1 , in which case their posterior belief $\Theta_{n}$ that the quantity of money is equal to $m+\Delta_{L}$ is

$$
\Theta_{n}=\frac{\left(1-m-\Delta_{L}\right) \Delta_{L}}{\left(1-m-\Delta_{L}\right) \Delta_{L}+\left(1-m-\Delta_{H}\right) \Delta_{H}} .
$$

There are also buyers at date 1 who received their unit of money at the end of date 0 . If $\Theta_{O}$ denote their posterior belief that the quantity of money in the economy is equal to $m+\Delta_{L}$, we obtain 


$$
\Theta_{o}=\frac{1-m-\Delta_{L}}{1-m-\Delta_{L}+1-m-\Delta_{H}} .
$$

Note that $\Theta_{o}>\Theta_{n}>\Theta_{s}$. Thus, buyers at date 1 carrying old money are more optimistic with respect to the value of money than buyers carrying new money. Moreover, date 1 sellers have an intermediate expectation with respect to the value of money at date 2 .

If buyers are constrained to offer a transfer $\tau \in[0,1]$, there exists an equilibrium where all buyers propose $\tau_{S}$, where

$$
q=\beta \tau_{s}\left[\Theta_{S} V_{1}\left(\Delta_{L}\right)+\left(1-\Theta_{S}\right) V_{1}\left(\Delta_{H}\right)\right],
$$

and $q$ is the quantity that the seller is committed to produce. Since

we obtain

$$
V_{1}\left(\Delta_{k}\right)=\frac{\left(1-m-\Delta_{k}\right)[u(q)-q]}{1-\beta},
$$

$$
\tau_{s}=\frac{q}{\beta\left[\Theta_{S} V_{1}\left(\Delta_{L}\right)+\left(1-\Theta_{s}\right) V_{1}\left(\Delta_{H}\right)\right]}=\frac{1-\beta}{\beta} \frac{q}{u(q)-q} \frac{1}{\widehat{\Theta}_{s}},
$$

where

$$
\widehat{\Theta}_{i} \equiv \Theta_{i}\left(1-m-\Delta_{L}\right)+\left(1-\Theta_{i}\right)\left(1-m-\Delta_{H}\right),
$$

and $i \in\{0, s, n\}$. Note that (4) implies $\tau_{s}<1$. The payoff of the buyer in the meeting with a seller is then given by

$$
\begin{aligned}
& u(q)+\beta\left(1-\tau_{S}\right)\left[\Theta_{i} V_{1}\left(\Delta_{L}\right)+\left(1-\Theta_{i}\right) V_{1}\left(\Delta_{H}\right)\right]= \\
& =u(q)+\beta\left(1-\tau_{s}\right) \widehat{\Theta}_{i} \frac{u(q)-q}{1-\beta},
\end{aligned}
$$

which can be rewritten as

$$
\begin{aligned}
& u(q)+\beta\left[1-\frac{1-\beta}{\beta} \frac{q}{u(q)-q^{*}} \frac{1}{\widehat{\Theta}_{s}}\right] \widehat{\Theta}_{i} \frac{u(q)-q}{1-\beta}= \\
& =u(q)+\widehat{\Theta}_{i}\left\{\frac{\beta}{1-\beta}[u(q)-q]-\frac{q}{\widehat{\Theta}_{s}}\right\} .
\end{aligned}
$$

We need to make sure that a buyer does not want to deviate from the offer $\tau_{s}$. Note that the only potentially profitable deviation involves no trade in period 1. The reasoning runs as follows. The buyer has no incentive to choose a higher transfer since this will not affect his current payoff (the seller always produces $q^{*}$ ) and it reduces his continuation payoff. In turn, if the buyer asks for a lower transfer, the seller may only be willing to accept such transfer if he believes that such an offer is coming from an optimistic buyer. Indeed, this belief increases his posterior that the quantity of money in the economy is $m+\Delta_{L}$, which compensates for the 
lower probability that money will be transferred. However, if the seller is willing to accept a lower transfer, it is also in the interest of the pessimistic buyer to offer a lower transfer. Thus, after observing a lower transfer, the seller must keep his original belief. This implies that the seller rejects the offer because it violates his incentive constraint.

Hence, since the only potentially profitable deviation involves no trade, the buyer will not deviate if and only if

$$
u(q)+\widehat{\Theta}_{i} \frac{\beta}{1-\beta}[u(q)-q]-\frac{\widehat{\Theta}_{i}}{\widehat{\Theta}_{s}} q \geq \widehat{\Theta}_{i} \frac{\beta}{1-\beta}[u(q)-q],
$$

that is

$$
\frac{u(q)}{q} \geq \frac{\widehat{\Theta}_{i}}{\widehat{\Theta}_{S}}
$$

Clearly, the strongest incentive to deviate comes from an optimistic buyer. Thus, we need

$$
\frac{u(q)}{q} \geq \frac{1-m-\left[\Theta_{o} \Delta_{L}+\left(1-\Theta_{o}\right) \Delta_{H}\right]}{1-m-\left[\Theta_{S} \Delta_{L}+\left(1-\Theta_{S}\right) \Delta_{H}\right]} .
$$

If $\Delta_{H}=\Delta_{L}$, the right-hand side of (5) is equal to 1 . Thus, there exists $\underline{\Delta}>0$ such that (5) holds whenever $\Delta_{H}-\Delta_{L} \leq \underline{\Delta}$. Proposition 1 summarizes the result.

Proposition 1. Let the set of possible offers be given by $\tau \in[0,1]$, and assume that $\Delta_{H}-\Delta_{L} \leq \underline{\Delta}$. There exists an equilibrium where the efficiency quantity $q^{*}$ is always produced in all trade meetings.

\subsubsection{Bargaining}

The set of possible offers is now given by

$$
\Gamma=\left\{(\tau, q) \mid \tau \in[0,1] \text { and } q \in \mathbb{R}^{+}\right\} .
$$

Our first result is that the any equilibrium is necessarily inefficient, in the sense that the quantity produced in some meetings at date 1 is different from $q^{*}$.

Proposition 2. There is no equilibrium in which the efficient quantity $q^{*}$ is produced in all meetings at date 1 .

Proof. The proof is by contradiction. Assume that there is an equilibrium where the efficient quantity is produced in all meetings at date 1 . Thus, it must be that all buyers propose the pair $\left(\tau_{s,} q^{*}\right)$, where

$$
\tau_{s}=\frac{1-\beta}{\beta} \frac{q^{*}}{u\left(q^{*}\right)-q^{*}} \frac{1}{\widehat{\Theta}_{s}} .
$$


In this case, the payoff to a buyer with posterior $i \in\{n, o\}$ is

$$
u\left(q^{*}\right)+\widehat{\Theta}_{i}\left[\frac{\beta}{1-\beta}\left[u\left(q^{*}\right)-q^{*}\right]-\frac{q^{*}}{\widehat{\Theta}_{s}}\right] .
$$

Consider now a deviation $\left(\tau_{s}-\epsilon, q^{*}-\delta\right)$ by an optimistic buyer. If the seller attaches probability one that the deviation indeed came from the optimistic buyer, his posterior $\Theta_{S}^{\text {old }}$ is

$$
\Theta_{S}^{\text {old }}=\frac{\left(1-m-\Delta_{L}\right)}{\left(1-m-\Delta_{L}\right)+\left(1-m-\Delta_{H}\right)} \equiv \Theta_{o} .
$$

This implies that

$$
q^{*}-\delta=\beta\left(\tau_{s}-\epsilon\right)\left[\Theta_{o} V_{1}\left(\Delta_{L}\right)+\left(1-\Theta_{o}\right) V_{1}\left(\Delta_{H}\right)\right]
$$

Since

$$
V_{1}\left(\Delta_{k}\right)=\frac{\left(1-m-\Delta_{k}\right)\left[u\left(q^{*}\right)-q^{*}\right]}{1-\beta},
$$

(6) can be rewritten as

$$
q^{*}-\delta=\beta\left(\tau_{s}-\epsilon\right) \widehat{\Theta}_{o} \frac{\left\lfloor u\left(q^{*}\right)-q^{*}\right\rfloor}{1-\beta} .
$$

Thus, the payoff of the buyer is

$$
u\left(q^{*}-\delta\right)+\beta\left[1-\left(\tau_{s}-\epsilon\right)\right] \widehat{\Theta}_{o} \frac{\left[u\left(q^{*}\right)-q^{*}\right]}{1-\beta},
$$

which can be rewritten as

$$
u\left(q^{*}-\delta\right)-\left(q^{*}-\delta\right)+\beta \widehat{\Theta}_{o} \frac{\left[u\left(q^{*}\right)-q^{*}\right]}{1-\beta} .
$$

The deviation is profitable if and only if

$$
\begin{aligned}
& u\left(q^{*}-\delta\right)-\left(q^{*}-\delta\right)+\beta \widehat{\Theta}_{o} \frac{\left[u\left(q^{*}\right)-q^{*}\right]}{1-\beta}> \\
& >u\left(q^{*}\right)+\widehat{\Theta}_{o}\left[\frac{\beta}{1-\beta}\left[u\left(q^{*}\right)-q^{*}\right]-\frac{q^{*}}{\widehat{\Theta}_{s}}\right],
\end{aligned}
$$

which can be rewritten as

$$
u\left(q^{*}\right)-u\left(q^{*}-\delta\right)<\frac{\widehat{\Theta}_{o}}{\widehat{\Theta}_{S}} q^{*}-\frac{\widehat{\Theta}_{o}}{\widehat{\Theta}_{o}}\left(q^{*}-\delta\right) .
$$

It remains to show that a pessimistic buyer has no incentive to make such a deviation. If he does, his payoff is

$$
u\left(q^{*}-\delta\right)+\beta[1-(\tau-\epsilon)] \widehat{\Theta}_{n} \frac{\left[u\left(q^{*}\right)-q^{*}\right]}{1-\beta}
$$


Substituting (7) in the above expression, we obtain

$$
u\left(q^{*}-\delta\right)-\frac{\widehat{\Theta}_{n}}{\widehat{\Theta}_{o}}\left(q^{*}-\delta\right)+\beta \widehat{\Theta}_{n} \frac{\left[u\left(q^{*}\right)-q^{*}\right]}{1-\beta} .
$$

The deviation is not profitable if and only if

$$
\begin{aligned}
& u\left(q^{*}\right)+\widehat{\Theta}_{n}\left[\frac{\beta}{1-\beta}\left[u\left(q^{*}\right)-q^{*}\right]-\frac{q^{*}}{\widehat{\Theta}_{s}}\right]> \\
& >u\left(q^{*}-\delta\right)-\frac{\widehat{\Theta}_{n}}{\widehat{\Theta}_{o}}\left(q^{*}-\delta\right)+\beta \widehat{\Theta}_{n} \frac{\left[u\left(q^{*}\right)-q^{*}\right]}{1-\beta},
\end{aligned}
$$

which can be rewritten as

$$
u\left(q^{*}\right)-u\left(q^{*}-\delta\right)>\frac{\widehat{\Theta}_{n}}{\widehat{\Theta}_{s}}\left(q^{*}\right)-\frac{\widehat{\Theta}_{n}}{\widehat{\Theta}_{o}}\left(q^{*}-\delta\right) .
$$

Note that, for any $\delta, \frac{\widehat{\Theta}_{o}}{\widehat{\Theta}_{S}}>1>\frac{q^{*}-\delta}{q^{*}}$ implies

$$
\frac{\widehat{\Theta}_{o}}{\widehat{\Theta}_{S}} q^{*}-\frac{\widehat{\Theta}_{o}}{\widehat{\Theta}_{o}}\left(q^{*}-\delta\right)>\frac{\widehat{\Theta}_{n}}{\widehat{\Theta}_{S}} q^{*}-\frac{\widehat{\Theta}_{n}}{\widehat{\Theta}_{o}}\left(q^{*}-\delta\right) .
$$

Then, let $\hat{\delta}$ be such that

$$
u\left(q^{*}\right)-u\left(q^{*}-\hat{\delta}\right)=\frac{\widehat{\Theta}_{n}}{\widehat{\Theta}_{s}} q^{*}-\frac{\widehat{\Theta}_{n}}{\widehat{\Theta}_{o}}\left(q^{*}-\hat{\delta}\right) .
$$

This value exists because, when $\delta=0$, the left hand side of (8) is smaller than the right hand side. In turn, when $\delta=q^{*}$, the left hand side of (8) is larger than than the right hand side. As a result, we have

$$
\begin{aligned}
& \frac{\widehat{\Theta}_{o}}{\widehat{\Theta}_{S}} q^{*}-\frac{\widehat{\Theta}_{o}}{\widehat{\Theta}_{o}}\left(q^{*}-\delta\right)>u\left(q^{*}\right)-u\left(q^{*}-\hat{\delta}\right)= \\
& =\frac{\widehat{\Theta}_{n}}{\widehat{\Theta}_{S}} q^{*}-\frac{\widehat{\Theta}_{n}}{\widehat{\Theta}_{o}}\left(q^{*}-\hat{\delta}\right) .
\end{aligned}
$$

In this case, an optimistic buyer has an incentive to deviate by choosing

$$
\left(\tau_{s}-\epsilon, q^{*}-\delta\right)=\left(\frac{(1-\beta)\left(q^{*}-\delta\right)}{\beta\left[u\left(q^{*}\right)-q^{*}\right] \widehat{\Theta}_{o}}, q^{*}-\hat{\delta}\right)
$$

But the pessimistic buyer has no incentive to do so. Finally, note that after observing such a deviation, the seller must attach probability one that the deviation came from the optimistic buyer. 
In the proof of Proposition 1, we used the Intuitive Criterion of Cho and Kreps (1987), a common refinement usually used in the literature of signaling games. It is a natural criterion in our framework. In our economy, buyers have different incentives to trade depending on their experience in the market. In particular, buyers with new money are more eager to transfer money in exchange for a larger quantity of output, since they are not very confident as to the future value of money in the economy. In the context of monetary models, the Intuitive Criterion is applied in a similar way by Nosal and Wallace (2004). They focus on the conditions under which a monetary equilibrium exists in an economy where counterfeit money can potentially circulate.

We have shown that an efficient equilibrium arises only if buyers are restricted to specify their offers in terms of a transfer of money, without explicitly stating the quantity of goods they want in exchange for the money. In this case, buyers are forced to choose the same action, irrespective of their private valuation of money. It turns out that, as soon as some additional channel of communication is present, the efficient equilibrium collapses.

In a sense, our result reverses the one in Berentsen et al. (2002). In their complete information model, the possibility of stating offers as a pair $(\tau, q)$ was beneficial as it avoided over production and allowed the efficient quantity to be produced in all meetings. In our model, there is no such benefit under complete information (dates $t>1$ ) because we are focusing on efficient equilibrium, where the quantity produced is always $q^{*}$. Moreover, there is a loss under incomplete information (date 1) because the possibility to communicate information by using more channels creates incentive for optimistic buyers to ask for a lower quantity of output.

We close our discussion by proving that there exists a separating equilibrium when buyers are allowed to propose a pair $(\tau, q)$. This equilibrium always reveals more information about the state of the economy but is always inefficient.

Proposition 3. There exists a separating equilibrium. In this equilibrium, the efficient quantity is traded in all dates $t>1$. In date 1 , the efficient quantity is produced in trade meetings with pessimistic buyers, but there is under production in trade meetings with optimistic buyers.

Proof. The behavior of buyers and sellers in all dates $t>1$ are exactly as in subsection 2.1. Consider now the behavior in date 1. Assume that a pessimist buyer asks for $\left(\tau_{n}, q^{*}\right)$, and that this offer is accepted by the seller. Thus,

$$
q^{*}=\beta \tau_{n}\left[\Theta_{n} V_{1}\left(\Delta_{L}\right)+\left(1-\Theta_{n}\right) V_{1}\left(\Delta_{H}\right)\right] .
$$

Since

$$
V_{1}\left(\Delta_{k}\right)=\frac{\left(1-m-\Delta_{k}\right)\left[u\left(q^{*}\right)-q^{*}\right]}{1-\beta},
$$


we obtain

$$
\tau_{n}=\frac{1-\beta}{\beta} \frac{q^{*}}{u\left(q^{*}\right)-q^{*}} \frac{1}{\widehat{\Theta}_{n}} .
$$

Moreover, the payoff of the pessimist buyer is

$$
u\left(q^{*}\right)-q^{*}+\beta \widehat{\Theta}_{n} \frac{\left[u\left(q^{*}\right)-q^{*}\right]}{1-\beta} .
$$

The problem of the optimist buyer is

$$
\max _{(\tau, q)}\left\{u(q)-\tau \widehat{\Theta}_{o} \frac{\beta}{1-\beta}\left[u\left(q^{*}\right)-q^{*}\right]\right\}
$$

subject to the seller's incentive constraint

$$
q=\tau \widehat{\Theta}_{o} \frac{\beta}{1-\beta}\left[u\left(q^{*}\right)-q^{*}\right],
$$

and the condition that the pessimist buyer has no incentive to mimic the choice of the optimist buyer, i.e.,

$$
u\left(q^{*}\right)-q^{*} \geq u(q)-\tau \widehat{\Theta}_{n} \frac{\beta}{1-\beta}\left[u\left(q^{*}\right)-q^{*}\right] .
$$

We can rewrite this problem as

$$
\max _{(\tau, q)}[u(q)-q]
$$

subject to the seller's incentive constraint

$$
u(q)-\frac{\widehat{\Theta}_{n}}{\widehat{\Theta}_{o}} q \leq u\left(q^{*}\right)-q^{*} .
$$

It must be the case that the constraint binds and we have

$$
u(q)-\frac{\widehat{\Theta}_{n}}{\widehat{\Theta}_{o}} q=u\left(q^{*}\right)-q^{*} .
$$

It remains to ensure that the optimist buyer has no incentive to mimic the behavior of the pessimist buyer. If he does so, he obtains

$$
u\left(q^{*}\right)-\frac{\widehat{\Theta}_{o}}{\widehat{\Theta}_{n}} q^{*}+\frac{\beta}{1-\beta} \widehat{\Theta}_{o}\left[u\left(q^{*}\right)-q^{*}\right] .
$$

Thus, we must have

$$
u(q)-q>u\left(q^{*}\right)-\frac{\widehat{\Theta}_{o}}{\widehat{\Theta}_{n}} q^{*} .
$$

Using (9), we can rewrite this last inequality as 


$$
\frac{\widehat{\Theta}_{o}}{\widehat{\Theta}_{n}}>\frac{q}{q^{*}}
$$

This is always satisfied because (9) implies $q<q^{*}$. Summarizing, there is a separating equilibrium where the pessimist buyer asks

$$
(\tau, q)=\left(\frac{1-\beta}{\beta} \frac{q^{*}}{u\left(q^{*}\right)-q^{*}} \frac{1}{\widehat{\Theta}_{n}}, q^{*}\right),
$$

and the optimist buyer asks

$$
(\tau, q)=\left(\frac{1-\beta}{\beta} \frac{q}{u\left(q^{*}\right)-q^{*}} \frac{1}{\widehat{\Theta}_{o}}, q \text { such that } u(q)-\frac{\widehat{\Theta}_{n}}{\widehat{\Theta}_{o}} q=u\left(q^{*}\right)-q^{*}\right) .
$$

This concludes our proof.

\section{Conclusion}

We have compared two trading protocols in markets with informational and search frictions. The first protocol, labeled price posting, delivers efficient quantities produced in all matches, but the second one, labeled bargaining, leads to faster learning because it provides more instruments for the agents to extract information in trade meetings.

Although in a stylized way, our model conveys the following message. In relatively stable markets (e.g., retail markets), price posting emerges as a prevailing trade mechanism. But if a market is characterized by a constant state of flux and uncertainty (e.g., the Federal Funds market), then buyers and sellers do better by participating in a series of bargaining sessions constantly updating their beliefs about the state of the market.

\section{References}

Afonso, G. and Lagos, R. (2015). Trade Dynamics in the Market for Federal Funds. Econometrica, 83(1), pp. 263-313.

Araujo, L. and Shevchenko, A. (2006). Price Dispersion, Information and Learning. Journal of Monetary Economics, 53(6), pp. 1197-1223.

Bartolini, L., Gudell, S., Hilton, S. and Schwarz, K. (2005). Intraday Trading in the Overnight Federal Funds Market. Current Issues in Economics and Finance, 11(11), pp. 70-94.

Berentsen, A., Molico, M. and Wright, R. (2002). Indivisibilities, Lotteries and Monetary Exchange. Journal of Economic Theory, 107, pp. 70-94.

Camera, G. and Delacroix, A. (2004). Trade Mechanism Selection in Markets with Frictions. Review of Economic Dynamics, 7, pp. 851-868. 
Cho, I. and Kreps, D. (1987). Signaling Games and Stable Equilibria. Quarterly Journal of Economics, 102, pp. 179-221.

Faig, M. and Li, Z. (2009). The Welfare Costs of Expected and Unexpected Inflation. Journal of Monetary Economics, 56(7), pp. 1004-1013.

Golosov, M. and Lucas, R. (2007). Menu Costs and Phillips Curves. Journal of Political Economy, 115(2), pp. 171-199.

Hu, T. and Wallace, N. (2016). Phillips Curve in a Matching Model. Working paper. Available at: https://taiweihu.weebly.com/uploads/6/3/1/9/631996/hu-wallacesubmission.pdf

Jones, L. and Manuelli, R. (2001). Volatile Policy and Private Information: The Case of Monetary Shocks. Journal of Economic Theory, 99, pp. 265-296.

Katzman, B., Kennan, J. and Wallace, N. (2003). Output and Price Level Effects of Monetary Uncertainty in a Matching Model. Journal of Economic Theory, 108, pp. 217-255.

Lucas, R.E. Jr. (1972). Expectations and the Neutrality of Money. Journal of Economic Theory, 4, pp. 103-124.

Nosal, E. and Wallace, N. (2007). A Model of (the Threat of) Counterfeiting. Journal of Monetary Economics, 54(4), pp. 994-1001.

Stigler, G. (1961). The Economics of Information. Journal of Political Economy, 69, pp. 21325.

Trejos, A. and Wright, R. (1995). Search, Bargaining, Money, and Prices. Journal of Political Economy, 103(1), pp. 118-141.

Wallace, N. (1997). Short-Run and Long-Run Effects of Changes in Money in a RandomMatching Model. Journal of Political Economy, 105(6), pp. 1293-1307. 